\title{
Again?!! The Emotional Experience of Social Notification Interruptions
}

\author{
Celeste Lyn Paul, Anita Komlodi, and Wayne Lutters \\ University of Maryland Baltimore County \\ 1000 Hilltop Circle, Baltimore, Maryland, United States \\ \{cpaul2, komlodi, lutters\}@umbc . edu
}

\begin{abstract}
This paper describes a post-hoc analysis of the relationship between the socialness of an interruptive notification and the emotional tone of the words used to describe the experience through a One-Word-Response (OWR). Out of the 89 responses analyzed, $73 \%$ of participants used emotional words to describe their notification experiences. There was a significant relationship between the emotional tone of a OWR response and the socialness of an interruptive notification experience and participants were 3.2 more likely to describe social interruptive notifications with positive words than negative words.
\end{abstract}

Keywords: Emotion, interruption, One-Word-Response, methodology, notification, user experience.

\section{Introduction}

Notifications are intended to be a useful service provided by an information technology to help users maintain awareness of events and information while they focus on other tasks. In the case of desktop-based notifications, this information can be delivered in the form of a message and displayed on a screen in a variety of ways - either by itself or in combination with an auditory or haptic cue-in order to gain the user's attention. These services can notify the user about new emails, keep track of calendar events, update chat availability status, and inform of new incoming messages from friends. Typically these notifications interrupt the user while engaged in other tasks, for example word processing or image editing.

Different types of notifications have different types of interruptive effects on the user. Interruptions could be beneficial by delivering anticipated information or they could have a negative effect by creating a distraction. Distractions or other negative experiences could affect the user performance and satisfaction of the user's current activity or of the notification system itself. Creating an effective notification system requires design trade-offs that balance user awareness with user distraction.

Social communication services actively connect people together through email, chat, and social networking. These services are constantly updated - delivering new information to subscribers all the time. As participation in social communication services increases, the amount of new information a user must be aware of increases. As a result, the number of notifications increases and so do the interruptions from those notifications. 
Previous work informs us when during an activity is best to interrupt users $[4,15]$, how to best design software to manage this $[3,13,14]$, and how to interrupt users in specialized domains [1,17]. The literature does not help us understand for what reasons users should be interrupted. Understanding "why" depends highly on understanding the notification and the context and there is little research in this area. We set out to investigate these contextual factors through an exploratory survey. The study asked participants to report a recent interruption experience and their reaction to it. Among other questions they were asked to describe the experience in one word.

Emotion is an important aspect of the user experience and influences how users understand, interpret, experience, and interact with technology [2,6]. However, emotion can be difficult to study because it is context dependent. There is no single method that is best for studying emotion and the best method is often determined by the scope of the study and type of data desired [10,12]. There are a range of methods available for studying emotion that include: using pictogram surveys such as the Selfassessment Manikin (SAM) [11] and Emocards [5], multimedia tools such as Experience Clip [16] and 3E [9] that allow participants to create media to express themselves, and physiological measurements such as EKG, heart rate, blood pressure, respiratory rate, and galvanic skin response [12]. The One-Word-Response technique described in this study (see Methodology) is most similar to the participant selfassessment methods used to study emotion.

\section{Methodology}

The purpose of the study described in this paper was to explore the interruptive notification experience with a focus on understanding the context of the experience and to help identify areas for further study. An interruptive notification is a notification that displays in a way that actively draws the user's attention. Emotion was discovered as an emergent factor during analysis which inspired further post-hoc analysis. This paper presents the results of the post-hoc analysis of the relationship between the emotional tone of words elicited from a One-Word-Response question and the socialness of the interruptive notifications collected from the main study.

The main study collected 139 responses regarding a participant's recent notification interruption experience using Amazon Mechanical Turk (AMT). AMT was used as a way to gain access to a more representative population sample and an alternative to the common approach of using college students. Participants were recruited and paid through the AMT system. Participation in the study was limited to participants in the United States to help control for English language skills, and was limited to participants who had at least a 95\% Human Intelligence Task (HIT) approval rating (the percentage of HITs correctly completed) in order to control for participants who are known to not follow instructions and provide low-quality responses.

The study instrument was a web-based form that asked participants to describe a recent interruptive notification experience through a combination of open-ended (text) and closed-ended (selection or scale) questions about the details of their experiences:

1. Describe the most recent pop-up notification you received (text)

2. Describe what you were doing at the time of the notification (text)

3. How long ago did you receive the notification? (selection) 
Table 1. Description of study rounds for data collection

\begin{tabular}{|c|c|c|c|c|}
\hline & Round 1 & Round 2 & Round 3 & Total \\
\hline All-Notifications & 20 & 20 & 20 & 60 \\
\hline Social-Notifications & 32 & 20 & 27 & 79 \\
\hline & & \multicolumn{2}{|c|}{ Total collected responses } & 139 \\
\hline & & \multicolumn{2}{|c|}{ Total valid responses } & 122 \\
\hline & \multicolumn{3}{|c|}{ Total OWR emotional tone responses } & 89 \\
\hline
\end{tabular}

4. Describe the type of notification message (e.g. New email message, Software updates available) (text)

5. What application or service did the notification come from (e.g. AOL Instant Messenger, Facebook, Windows Updates) (text)

6. Did you feel that you needed to take action or respond to the notification? (selection + text)

7. Did you take action or respond to the notification? (selection + text)

8. Rate the notification based on the following qualities: Important, Interesting, Urgent, Useful, Valuable (scale)

9. Which of these qualities is the most important to you when receiving any notification? (selection)

10. How often would you want notifications like the one you received in the future? (selection)

11. Using one word, how would you describe the notification you received? (text)

The main study was conducted in two parts. The All-Notifications part asked participants to respond based on any recent interruptive notification experience. The Social-Notifications part asked participants to respond based only a recent social-only interruptive notification experience. The study instruments were the same for both parts except that the instructions for the Social-Notifications part specified social-only interruptive notification experiences. Each study part was conducted over three rounds of AMT studies until 20 responses that followed instructions were collected. Separating the studies into multiple rounds is best for AMT studies because newer studies requiring fewer responses have better response rates than older studies requiring many responses. Table 1 describes how the study rounds were conducted.

One-Word-Response. One-Word-Response (OWR) is a word association technique that is a short, direct question that requests the participant to respond with a single word. Word association is an elicitation technique that aims to get an immediate reaction rather than a thought-out response. OWR differs from other survey question types in that it is a simple question with a simple response and requires no deliberation to respond to. This question type is common, but we formalize the technique in this study. Although the study instructions asked participants to provide a "description", to our surprise, many of them gave emotional words as responses. We report the analysis of these emotional responses in this paper. Word association techniques can be susceptible to priming [8], a memory effect due to a previous influence or exposure, and so the responses must be considered within the context of other influences in the study. The OWR in this study was worded as: "Using one word, how would you describe the notification?" 
Table 2. Positive and negative OWR responses with frequency

\begin{tabular}{|c|c|c|}
\hline \multicolumn{2}{|c|}{ Positive Words } & \multirow{2}{*}{ Negative Words } \\
\hline - $\quad$ Informative (9) & - $\quad$ Easy (1) & \\
\hline - $\quad$ Useful (7) & - $\quad$ Fast (1) & - Unimportant (3) \\
\hline - $\quad$ Helpful (6) & - $\quad$ Fun (1) & - $\quad$ Boring (2) \\
\hline - Important (6) & - $\quad$ Good (1) & - $\quad$ Irritating (2) \\
\hline - $\quad$ Exciting (4) & - $\quad$ Great (1) & - $\quad$ Again?!! (1) \\
\hline - $\quad \operatorname{Cool}(3)$ & - $\quad$ Happy (1) & - $\quad$ Bad timing (1) \\
\hline - Convenient (2) & - Humorous (1) & - $\quad$ Bothered (1) \\
\hline - $\quad$ Effective (2) & - $\quad$ Necessary (1) & - $\quad$ Distracting (1) \\
\hline - $\quad$ Efficient (2) & - $\quad$ Polite (1) & - $\quad$ Forgettable (1) \\
\hline - $\quad$ Interesting (2) & - $\quad$ Remindful (1) & - $\quad$ Pestering (1) \\
\hline - $\quad$ Pleasant (2) & - $\quad$ Simple (1) & - $\quad$ Time waste (1) \\
\hline - $\quad$ Discreet (1) & - $\quad$ Thankful (1) & $\begin{array}{ll}\text { - } & \text { Untimely (1) } \\
\text { - } & \text { Useless(1) }\end{array}$ \\
\hline
\end{tabular}

Data Quality. Responses were reviewed to ensure the collected data was about a recent notification experience and not an experience similar to but not the same as interacting with a notification. Each response was evaluated based on two rules: First, if the notification was part of the main task and required a response before the user could continue this main task (For example, a browser security alert requiring the user to accept a cookie before continuing); and second, if the response was a web-based pop-up (For example an advertisement). Responses that met a rule were discarded since participants did not follow instructions. Of the 139 responses collected, 17 responses met one of these rules and were discarded for a total of 122 valid responses collected.

Data Coding. Coding rules for notification socialness were developed ad-hoc as part of the study design while OWR emotion coding rules were developed post-hoc in response to the need for additional analysis.

Notification Socialness. Participants were asked to describe a recent notification experience including what they were doing at the time of the notification and details of the notification. These details contained information about the application or service that sent the notification and what the notification was about, e.g. "An incoming message from a friend on Facebook" and "A notification that I needed to update my anti-virus software". From these descriptions, responses were coded as either social or non-social. If the notification came from a social application or service it was coded as a social notification. If the notification was not a social application or service it was coded as non-social notification.

OWR Emotion. OWR responses were coded based on an emotional dictionary that defined the positive or negative tone of the emotion [7]. OWRs were coded either positive for words with a positive emotional tone, negative for words with a negative emotional tone, or descriptive for words that had no emotional tone and simply described the experience. Words that had an unclear emotional tone were further investigated by evaluating the context of the open-ended responses. For example, two cases of "reminder" were clearly descriptive, while one case of "remindful" was clearly a positive word when the context of the open-ended responses considered. 
Table 3. Statistical analysis of OWR emotion x notification socialness

\begin{tabular}{lccccccc}
\hline \multirow{2}{*}{$\begin{array}{c}\text { Notification } \\
\text { Socialness }\end{array}$} & \multicolumn{3}{c}{ OWR Emotion } & \multicolumn{2}{c}{ Chi-square } & \multicolumn{2}{c}{ Phi } \\
\cline { 2 - 7 } & Positive & Negative & Total & Value $(d f)$ & Sig. & Value & Sig. \\
\hline Social & 35 & 10 & 45 & & & & \\
Non-social & 23 & 21 & 44 & $6.376(1)$ & .012 & 0.268 & .012 \\
Total & 58 & 31 & 89 & & & & \\
\hline
\end{tabular}

\section{Results and Discussion}

Even though the OWR question asked participants for a "description" of their experiences, most responses contained emotion. Of the 122 participant responses, 89 words $(73 \%)$ had a positive or negative emotional tone with 58 positive words (24 unique) and 31 negative words (13 unique). "Annoying" was the most popular negative word ( $n=15,48 \%$ of all negative words). There was no single popular positive word, with the top five positive words as "informative" ( $n=9,15 \%$ of all positive words), "useful" $(n=7,12 \%)$, "helpful" ( $n=6,10 \%)$, "important" $(n=6,10 \%)$, and "exciting" $(n=4,7 \%)$. Table 2 lists the positive and negative OWR responses. The prevalence of emotion suggests that the interruptive notification experience had a significant emotional effect on participants such that their reactions to the interruptive notifications were emotional. Perhaps participants found it easier to draw on an emotional word to describe their experience, which supports design literature that includes emotion as part of the interaction experience.

A Chi-square Test of Independence showed a significant relationship between the OWR emotional tone (positive/negative) and socialness of the interruptive notification (social/non-social) $(6.376, \mathrm{df}=1, \mathrm{p}=.012)$. A Phi coefficient showed a significant weak relationship between emotional tone and socialness $(r=.268, p=.012)$. A theta odds ratio showed that social interruptive notification experiences were 3.2 times more likely to be described with a positive OWR word than a non-social interruptive notification. Table 3 provides a summary of statistical analysis.

Social Interruptive Notifications. Notifications from Facebook were the most frequently reported source of social interruptive notifications $(n=31)$. These included interruptive notifications from all features of Facebook including chat, new mail messages, and other notices. Email (Gmail, Inbox.com, Outlook, Thunderbird, Yahoo!, but does not include Facebook messages) was the second most reported source of social interruptive notifications $(n=18)$, followed by chat (AOL Instant Messenger and MSN Messenger, not including Facebook chat, n=5). More positive words used to describe social interruptive notifications than negative words. Of the 45 social interruptive notification experiences, 35 were described using a positive emotional word compared to 10 that were described using a negative emotional word. Many participants expressed the social benefits of social interruptive notifications, as one participant stated, "I like knowing when someone quotes me [on a website]" (OWR "informative"). Another participant described a social obligation, "I didn't want to make my friend wait; [I responded immediately because] it was polite" (OWR "humorous"). These results suggest that social interruptive notifications are likely to be a positive experience. 
Non-social Interruptive Notifications. Software and security updates were the most common non-social interruptive notifications. Operating system updates from Windows (versions XP, Vista, and 7) and Mac OS X as the most frequently reported source of nonsocial interruptive notifications $(n=18)$. Security software (Avast, AVG, Immunet, Kaspersky, McAfee, Microsoft Security, Norton) were a close second ( $\mathrm{n}=15)$, followed by various software-related update services (Adobe $n=7, H P n=4$, Java $n=4$ ). However, there was no difference in the ratio of positive or negative words used to describe nonsocial interruptive notifications. Of the 44 non-social interruptive notification experiences described, 23 were described with positive words and 21 were described with negative words.

A further look into the experience of non-social interruptive notifications revealed mixed feelings about receiving notifications about software updates and security services. Some participants did not mind non-social notifications because, "It is always good to know your virus protection is working," (OWR "great"). Other participants expressed dislike because, "They interfere with what I was currently doing," (OWR "irritating"). Other factors may influence why one interruptive notification can be a better experience than another. For example, frequency of nonsocial interruptive notifications may be an important contextual factor. As one participant explained, "It's important to know that everything is working okay, but maybe not everyday," (OWR "boring"). Another participant stated, "The less often I get [notifications], the more likely I am to listen to them," (OWR "annoying"). This suggests two things about non-social interruptive notifications. First, that non-social interruptive notifications may be an emotional experience but not an overwhelmingly positive or negative one. Second, that non-social interruptive notifications may have additional factors that influence the overall experience; that is to say, context matters more for understanding the non-social interruptive notification experience.

\subsection{Study Limitations}

The purpose of the main study described in the Methodology was to collect information about recent experiences of interruptive notifications. The nature of our methodology may have resulted in participants to recall rather than to report their experiences. A study design with a different methodology could better support reporting and result in different OWR responses or different emotional content.

As previously stated in the Methodology, emotion was not a planned study factor. Due to our main study methodology and the post-hoc nature of our analysis, we were unable to capture a baseline of participants' emotional state before and after the interruptive notification experience. This type of comparison is common in emotional design research.

Finally, while the OWR and other study quests were descriptive in the way they were presented to participants, there was possible evidence of word priming. Some of the qualities participants were asked to rate in the main study appeared in the OWR responses as emotional words (e.g. useful and interesting). However, priming is not always s a negative effect and could be used as a methodological strategy. For example, in order to control context of responses, participants could be primed in a way to influence the scope of how they respond. 


\section{Conclusions}

We found a significant relationship (Chi-square 6.376, $\mathrm{df}=1, \mathrm{p}=.012$ ) between OWR emotional tone (positive/negative) and the socialness of an interruptive notification (social/non-social). Although the overall strength of the relationship was weak (Phi $\mathrm{r}=.268, \mathrm{p}=.012$ ), a positive word was more likely (theta odds ratio, 3.2) to be used to describe a social interruptive notification experience than a negative word. Social interruptive notifications stand out as particularly interesting results since many users' computing experiences are shifting from a work-related to more a social environment. Our results are important for two reasons. First, our results identify emotion as an important factor in the interruptive notification experience. Second, our results reinforces existing literature on emotion as an important factor in interaction design.

While socialness was planned as a factor to further investigate, the results presented in this paper has also lead us to include emotion as a factor to examine in any future work. We plan to continue investigating the contextual factors of the interruptive notification experience. A future field study will follow a small group of users who know and interact with each other over social communication tools. The primary research method will be a software-supported diary study with secondary methods to log user and system behavior. The diary study is unique in that the software will help negotiate common challenges found in diary study methodology such as consistency in event sampling, consistency in participant data entry, and consistency in participation over time.

\section{References}

1. Avrahami, D., Fussell, S.R., Hudson, S.E.: IM Waiting: Timing and Responsiveness in Semi-Synchronous Communication. In: Proc. ACM CSCW 2008, pp. 285-294 (2008)

2. Boehner, K., DePaula, R., Dourish, P., Sengers, P.: How Emotion is Made and Measured. Int. J. of Human-Computer Studies 65(4), 275-291 (2007)

3. Cadiz, J.J., Venolia, G., Jancke, G., Gupta, A.: Designing and Deploying an Information Awareness Interface. In: Proc. ACM CSCW 2002, pp. 314-323 (2002)

4. Czerwinski, M., Horvitz, E., Wilhite, S.: A Diary Study of Task Switching and Interruptions. In: Proc. ACM CHI 2004, pp. 175-182 (2004)

5. Desmet, P.M.A., Overbeeke, C.J., Tax, S.J.E.T.: Designing products with added emotional value; development and application of an approach for research through design. The Design Journal 4(1), 32-47 (2001)

6. Forlizzi, J., Battarbee, K.: Understanding Experience in Interactive Systems. In: Proc. Designing Interactive Systems 2004, pp. 261-268 (2004)

7. Hein, S.: Feeling Words List (2010), http: / /www . eqi . org/ (retrieved April 2011)

8. Hines, D., Czerwinski, M., Sawyer, P.K., Dwyer, M.: Automatic Semantic Priming: Effect of Category Exemplar Level and Word Association Level. J. Exp. Psych.: Human Perception and Performance 12(3), 370-379 (1986)

9. Isomursu, M., Kuutti, K., Vainamo, S.: Experience clip: method for user participation and evaluation of mobile concepts. In: Proc. Participatory Design Conference, pp. 83-92 (2004) 
10. Isomursu, M., Tahti, M., Vainamo, S., Kuutti, K.: Experimental evaluation of five methods for collecting emotions in field settings with mobile applications. Int. J. HumanComputer Studies 65, 404-418 (2007)

11. Lang, P.J.: Behavioral treatment and bio-behavioral assessment: computer applications. In: Sidowski, J.B., Johnson, J.H., Williams, T.A. (eds.) Technology in Mental Health Care Delivery Systems, pp. 119-139. Albex, Norwood (1980)

12. Lopatovska, I.: Researching Emotion: Challenges and Solutions. In: Proc. iConference 2011, pp. 225-229 (2011)

13. Mankoff, J., Dey, A.K., Hsieh, G., Kientz, J., Lederer, S., Ames, M.: Heuristic Evaluation of Ambient Displays. In: Proc. ACM CHI 2003, pp. 169-176 (2003)

14. Matthews, T., Dey, A.K., Mankoff, J., Carter, S., Rattenbury, T.: A Toolkit for Managing User Attention in Peripheral Displays. In: Proc. ACM UIST 2004, pp. 247-256 (2004)

15. Miyata, Y., Norman, D.A.: Psychological Issues in Support of Multiple Activities. In: Norman, D.A., Draper, S.W. (eds.) User Centered Systems Design: New Perspectives on Human-Computer Interaction, pp. 265-284. Lawrence Erlbaum Associates, Mahwah (1986)

16. Tahti, M., Arhippainen, L.: A Proposal of collecting Emotions and Experiences. In: Interactive Experiences in HCI, vol. (2), pp. 195-198 (2004)

17. Vastenburg, M.J., Keyson, D.V., de Ridder, H.: Considerate home notification systems: a field study of acceptability of notifications in the home. Personal and Ubiquitous Computing 12, 555-566 (2008) 